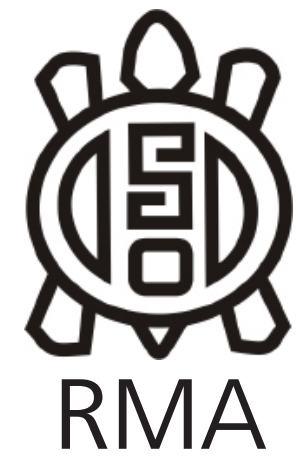

Antropología Biológica

\section{Análisis comparativo de fórmulas de estimación de la talla en restos humanos de Patagonia Austral. Primeros resultados}

Comparative analysis of stature estimation formulas in human remains of Southern Patagonia. First results

Rodrigo Zuñiga Thayer* y Jorge Suby**

*CONICET, Instituto Multidisciplinario de Historia y Ciencias Humanas, Buenos Aires, Argentina. Grupo de Investigación en Bioarqueología. E-mail: rzunigat89@gmail.com

**CONICET, Instituto de Investigaciones Arqueológicas y Paleontológicas del Cuaternario Pampeano, Facultad de Ciencias Sociales, Universidad Nacional del Centro de la Provincia de Buenos Aires, Quequén, Argentina. Grupo de Investigación en Bioarqueología. E-mail: jasuby@conicet.gov.ar

\begin{abstract}
Resumen
La talla en restos humanos de poblaciones arqueológicas representa un importante indicador general de la salud. Los enfoques para la estimación de talla son el anatómico y el matemático. El anatómico es considerado más exacto y no conlleva problemas de variabilidad interpoblacional, aunque su empleo se encuentra limitado por la necesidad de contar con una alta completitud esqueletal. El enfoque matemático, en cambio, utiliza ecuaciones de regresión que requieren la longitud de uno o más huesos largos. Sin embargo, se ha detectado alta variación en la relación entre la estatura real y los segmentos utilizados para estimarla. En Patagonia Austral, no existen fórmulas específicas para estas poblaciones, lo cual resulta en una baja comparabilidad de resultados y dificulta su selección al momento de estimar la talla. El objetivo de este trabajo es identificar qué fórmulas predicen con mayor exactitud la talla en relación con las estimaciones del método anatómico. Los resultados indican que la mayoría de las fórmulas matemáticas proporcionan resultados similares a los resultados obtenidos con la fórmula anatómica. Además, para ambos sexos las fórmulas con mayor coeficiente de determinación tienden a agruparse de acuerdo con el o los huesos requeridos. Las estimaciones de talla más exactas corresponden principalmente a aquellas que emplean el fémur.
\end{abstract}

Palabras clave: Bioarqueología; Cazadores-recolectores; Estatura; Salud; Metodología.

\begin{abstract}
Human stature in archaeological remains is an important indicator of general health in past human population. Anatomical and mathematical methods were proposed to reconstruction of stature. The anatomical method is considered more accurate although its use is limited by the high skeletal completeness required. The mathematical approach uses regression equations that require the length of one or more long bones, but high variation in the relationship between real height and the segments used to estimate it has been detected. There are no specific formulas for stature estimation from skeletal remains of Southern Patagonia. The objective of this work is to identify which mathematical formulas predict height more accurately in relation to the estimates of the anatomical method. Results show that most of the mathematical formulas are very similar to the results obtained from the anatomical formula. For both sexes, the formulas with the highest coefficients of determination are grouped according to the required bone. The most exact stature formulas correspond mainly to those that use the femur.
\end{abstract}

Keywords: Bioarchaeology; Hunter-gatherers; Stature; Health; Methodology.

La talla humana ha sido descripta como una característica biológica dinámica, determinada por una compleja red de interacciones entre el potencial genético y las condiciones de salud y nutrición (King y Ulijaszek, 1999; Vercellotti et al., 2014). Durante el periodo de crecimiento y desarrollo de un individuo los principales factores ambientales que pueden afectar la talla alcanzada en la adultez son las condiciones climáticas, la dieta, el estatus socioeconómico y algunas enfermedades (Bogin, 1999; Bogin y Keep, 1999; Komlos, 1998; Komlos y Lauderdale, 2007; Neves y 
Costa, 1998; Tanner, 1987). Aunque hasta el momento no ha sido posible determinar la influencia específica de cada factor, la talla estimada a partir de restos arqueológicos es considerada en bioarqueología como un indicador general de salud de poblaciones antiguas (Hoppa y Fitzgerald, 1999; Larsen, 1997; Saunders y Hoppa, 1993; Vercellotti et al., 2014).

La estimación de talla a partir de restos humanos se ha utilizado desde hace al menos un siglo (Dwight, 1894; Pearson, 1899; Rollet, 1888) a través de dos enfoques: el anatómico y el matemático (Lundy, 1985). El enfoque anatómico consiste en estimar la talla a partir de la sumatoria de las longitudes del fémur, de la tibia, de la altura del astrágalo y calcáneo, de los cuerpos vertebrales y del cráneo, junto con un factor de corrección para los tejidos blandos ausentes en huesos secos (Lundy, 1985). El desarrollo principal de este enfoque es atribuido al método propuesto por Fully (1956), el cual fue revisado y modificado posteriormente por Raxter et al. (2006). Varios autores consideran que este método como el más exacto, ya que tiene en cuenta a todas las porciones óseas y blandas que contribuyen en la talla real (Lundy, 1985; Olivier, 1969; Ousley, 1995; Raxter et al., 2006). Sin embargo, el empleo de este método está con frecuencia limitado por la necesidad de contar con una muy alta completitud esqueletal, no siempre disponible, particularmente en contextos arqueológicos (Lundy, 1985).

El enfoque matemático, por otra parte, utiliza ecuaciones de regresión basadas en la asociación de la longitud de uno o más elementos óseos con la talla. Con este objetivo, se han propuesto fórmulas de regresión construidas a partir de conjuntos de restos humanos identificados con información real de la talla de los individuos vivos (e.g. Genovés, 1967; Trotter y Glesser, 1952) o a partir de colecciones de restos humanos sin datos de talla real (Auerbach y Ruff, 2010; Béguelin, 2011). Las fórmulas de regresión resultan las más empleadas en general. Sin embargo, se ha destacado la alta variabilidad interpoblacional que presentan (Holliday, 1997; Holliday y Ruff, 1997; Stinson, 1990), por lo que la elección de las fórmulas adecuadas para estimar la talla en una muestra particular resulta de gran importancia.

Las fórmulas más difundidas han sido construidas a partir de muestras de poblaciones "blancas" y "negras" de América del Norte que conforman la Smithsonian Terry Collection de Estados Unidos (Trotter y Gleser, 1952), y de Mesoamérica, desarrolladas a partir de cadáveres de individuos mexicanos con ancestría indígena (Genovés, 1967). En todos los casos, las fórmulas son diferentes para cada sexo, contemplando así el dimorfismo sexual. En América del Sur han sido desarrolladas ecuaciones específicas para la estimación de talla en esqueletos de poblaciones andinas de costa y media altura (Pomeroy y Stock, 2012). En el caso del sur de Sudamérica, Béguelin
(2011) propuso fórmulas de regresión a partir de la estimación de la talla según Raxter et al. (2006) para individuos masculinos de Patagonia Central, aunque en este último caso se desconoce la talla real de los individuos que componen la muestra.

En Patagonia Austral, la región del continente comprendida al sur de la latitud $50^{\circ} \mathrm{S}$, las estimaciones y evaluaciones de la talla de individuos de poblaciones del pasado fueron exploradas en algunos casos a partir de individuos vivos y en otras a partir de restos humanos. Entre los primeros, Gusinde (1989) tomó mediciones somatométricas, incluyendo la talla, de 106 individuos (45 Selk'nam, 30 Yamana y 31 Halakwulup), entre los años 1919 y 1924. Esta información fue analizada posteriormente por Varela et al. (1993-94), encontrando que la talla es una de las características con mayor variabilidad entre los tres grupos, con un "gradiente de mayor a menor contextura física desde Selk'nam a Yamana y Halakwulup" (Varela, Cocilovo y Guichón, 1993-94:203), lo que fue sugerido como evidencia de una mayor asociación biológica entre los últimos dos grupos con respecto al primero. Recientemente estos datos fueron confirmados y fue determinado el efecto de la distancia espacial entre los grupos, es decir existió un mayor parentesco entre Selk'nam-Yámana y Yámana-Halakwulup, mientras que entre Selk'nam y Halakwulup las relaciones parentales fueron menos frecuentes (Cocilovo et al., 2019). Los mismos datos tomados por Gusinde (1989) fueron analizados por Soto-Heim (2001), e interpretados como evidencias no solo de factores ambientales, sino también de posibles variaciones nutricionales y actividad física.

En cuanto a estimaciones de la talla en restos humanos de las poblaciones antiguas de Patagonia Austral, Hernández et al. (1998) analizaron 25 esqueletos resguardados en el Instituto de la Patagonia (Universidad de Magallanes, Punta Arenas, Chile). Según estos autores, los individuos adscriptos a la etnia Halakwalup mostraron mayor talla que los Selk'nam, empleando fórmulas de regresión propuestas por Trotter y Gleser (1952) para mongoloides y Feldesman y Fountain (1996) a partir de mediciones del fémur. Estos resultados serían contradictorios con los reportados por Gusinde (1989) y luego interpretados por Varela et al. (1993-94) Soto-Heim (2001) y Cocilovo et al. (2019), aunque en este caso podrían existir sesgos producto de la asignación étnica a los esqueletos analizados. En efecto, más recientemente AlfonsoDurruty et al. (2017) realizaron estimaciones de talla en 51 esqueletos resguardados en la misma institución, cuyos resultados sugieren tendencias contrarias a las expuestas por Hernández et al. (1998). De acuerdo a sus resultados, los individuos asociados a economías terrestres muestran una mayor talla que los asociados a económicas marítimas, con valores intermedios para aquellos con dietas mixtas, y con un gradiente fenotípico de este a oeste. Estos resultados fueron interpretados 
como posible evidencia de influencia ambiental sobre la talla, especialmente producida por la temperatura y la humedad, y también como evidencia de la adaptación de las poblaciones humanas a esas condiciones. En ese trabajo, Alfonso-Durruty et al. (2017) seleccionaron fórmulas de regresión propuestas por Auerbach y Ruff (2010) para poblaciones de climas templados de América del Norte para mediciones del fémur, la tibia y la sumatoria de ambos. Esta selección se realizó considerando la similitud entre el índice crural (longitud femoral/longitud tibial), entre los individuos analizados y los de esa región de América del Norte (Alfonso-Durruty et al., 2017; Auerbach y Ruff, 2010). Además, estos autores emplearon las fórmulas de regresión propuestas por Béguelin (2011) para poblaciones de Patagonia Central.

Hasta el momento los trabajos que analizaron la talla a partir de restos humanos de Patagonia Austral - al no contar con fórmulas específicas para esas poblaciones - seleccionaron en cada caso fórmulas de regresión diferentes. Esto podría resultar en la baja comparabilidad de los resultados y en las dificultades para seleccionar una fórmula de regresión, al momento de analizar la talla de uno o más individuos de esta región. En consecuencia, el objetivo de este trabajo es identificar qué fórmulas de las propuestas por Trotter y Gleser (1952), Genovés (1967) y Béguelin (2011) predicen con mayor exactitud la talla en relación con las estimaciones del método de Fully (Raxter et al., 2006) en restos humanos de Patagonia Austral.

\section{Materiales y métodos}

Con el fin de analizar la concordancia entre estimaciones de la talla entre fórmulas de regresión, se seleccionaron individuos provenientes de Patagonia Austral (territorio sudamericano al Sur de la latitud $50^{\circ} \mathrm{S}$ ) de ambos sexos cuya estimación de edad al momento de la muerte fuera mayor a 18 años y que tuvieran presentes los elementos necesarios para estimar la talla según el método propuesto por Fully (Raxter et al., 2006) (i.e. cráneo, cuerpos vertebrales, sacro, fémur, tibia, calcáneo y astrágalo), basado en la suma de los segmentos óseos que contribuyen a la longitud vertical del esqueleto y un posterior ajuste por el tejido blando. Los individuos subadultos no fueron tenidos en cuenta dado que no han completado su crecimiento. Se tuvo acceso a individuos resguardados en instituciones de Argentina, que incluyeron al IMHICIHU-CONICET (Buenos Aires), al Museo del Fin del Mundo (Ushuaia), al CADIC-CONICET y al Laboratorio de Ecología Evolutiva Humana (UNICENQuequén).

El sexo de los esqueletos analizados fue estimado de acuerdo con las estructuras dimórficas de la pelvis y el cráneo, según los métodos propuestos por Buikstra y Ubelaker (1994). La edad de muerte fue estimada a partir del análisis de los cambios morfológicos de la superficie auricular (Lovejoy et al., 1985) y de la sínfisis púbica (Brooks y Suchey, 1990; Todd, 1921a, 1921b), siendo asignada luego a las categorías adultos jóvenes (18-34,9 años), adultos medios (35-49,9 años) y adultos mayores (+50 años). Luego de esta selección se contó con siete individuos, tres $(42,9 \%)$ de sexo femenino y cuatro $(57,1 \%)$ de sexo masculino (Tabla 1$)$. Cuatro de los individuos analizados son adultos jóvenes, mientras que 3 son adultos medios. Además, uno de ellos proviene de la provincia de Santa Cruz (Argentina), mientras que 4 fueron recuperados en sitios del norte de Tierra del Fuego y los 2 restantes en el sur de Tierra del Fuego.

La medición de las longitudes máximas de cada elemento fue tomada con una tabla osteométrica $(1 \mathrm{~mm}$ de precisión). En los individuos en los cuales estuvieran ausentes hasta un máximo de tres vertebras no consecutivas, se estimó la altura máxima de las vértebras ausentes como la media entre la altura de las vértebras adyacentes, siguiendo a Auerbach (2011). Esta corrección fue necesaria en sólo uno de los individuos analizados, en el cual no se encontraba presente la vértebra D6 (MFM -2670).

Para determinar la exactitud de las predicciones propuestas por Trotter y Gleser (1952), Genovés (1967)

Tabla 1. Individuos de Patagonia Austral seleccionados para estimar la talla a partir del método anatómico de Fully (1956). Referencias: SCruz: Santa Cruz; NTdF: Norte de Tierra del Fuego; STdF: Sur de Tierra del Fuego; M: Masculino; F: Femenino; AJ: Adulto Joven; AM: Adulto Medio; ND: No disponible.

Table 1. Skeletons from Southern Patagonia selected to estimate stature from Fully's anatomical revised method (Raxter et al 2006) References: SCruz: Santa Cruz; NTdF: Northern Tierra del Fuego; STdF: Southern Tierra del Fuego; M: Male; F: Female; AJ: Young adult; AM: Middle adult; ND: No data available.

\begin{tabular}{|l|l|l|l|c|l|}
\hline \multicolumn{1}{|c|}{ Individuo } & \multicolumn{1}{|c|}{ Región } & Sexo & \multicolumn{1}{|c|}{ Edad } & $\begin{array}{c}\text { Cronología } \\
\left({ }^{14} \mathrm{C} \text { años AP }\right)\end{array}$ & \multicolumn{1}{c|}{ Referencia } \\
\hline Orejas de Burro 2 & SCruz & M & AM & $3565 \pm 45$ & L'Heureux y Barberena, 2008 \\
\hline Las Mandibulas 2 & NTdF & M & AJ & Contacto & Guichón et al., 2000 \\
\hline Puesto Pescador 1 & NTdF & M & AJ & $335 \pm 35$ & Suby et al., 2008 \\
\hline La Arcillosa 2 & NTdF & F & AJ & $5208 \pm 58$ & Santiago et al., 2011 \\
\hline MFM-2667 & NTdF & M & AM & ND & Presente estudio \\
\hline Paiashauaia 1 & STdF & F & AM & $1504 \pm 46$ & Suby et al., 2011 \\
\hline MFM-2670 & STdF & F & AJ & ND & Presente estudio \\
\hline
\end{tabular}




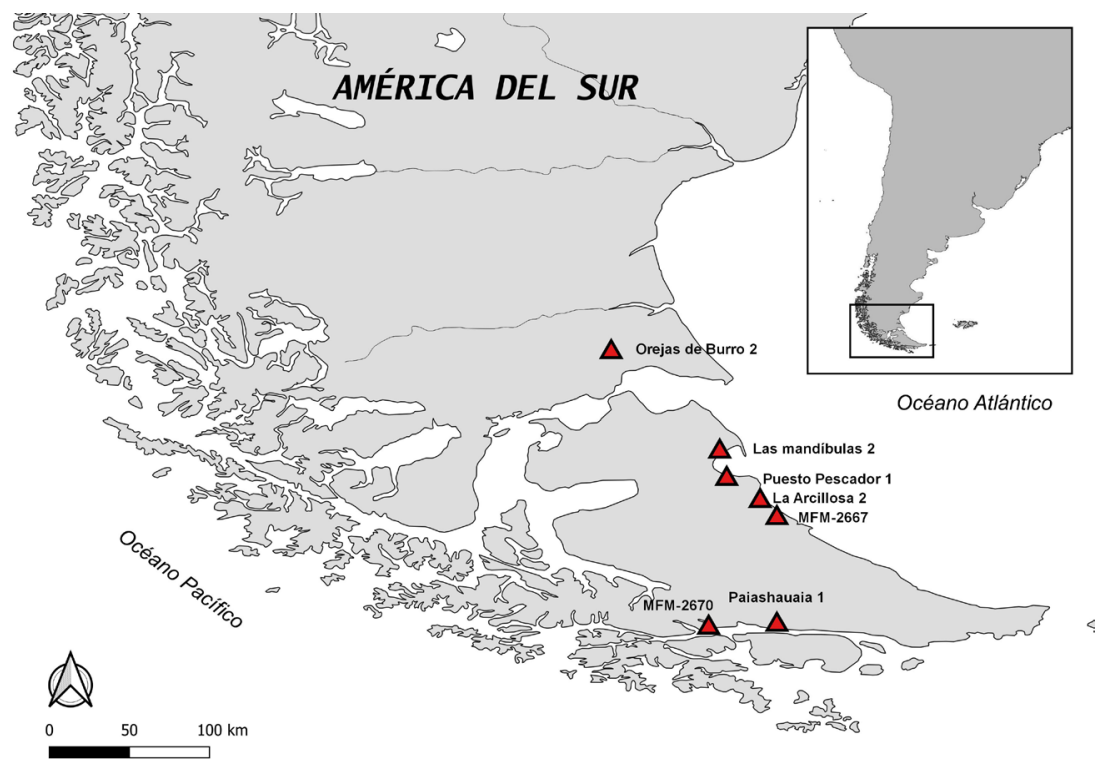

Figura 1. Localización de los sitios arqueológicos de los cuales provienen los restos humanos analizados.

Figure 1. Location of the archaeological sites where the skeletons included in this paper were recovered.

Tabla 2. Fórmulas de regresión (en $\mathrm{cm}$ ) empleadas sobre los individuos estudiados.

Table 2. Regression formulas (in $\mathrm{cm}$ ) used on the studied individuals

\begin{tabular}{|c|c|}
\hline \multicolumn{2}{|r|}{ Fórmula Anatómica de Fully (Raxter, 2006) } \\
\hline \multicolumn{2}{|r|}{ talla $=1,009 \times$ altura esqueletal $+11,7$} \\
\hline \multicolumn{2}{|r|}{ Fórmulas De Regresión Para Poblaciones de Patagonia Central (Béguelin, 2011) } \\
\hline \multicolumn{2}{|r|}{ Fémur: talla $=69,38+2,21 \times$ Long. Fémur $\pm 3,32$} \\
\hline \multicolumn{2}{|r|}{ Tibia: talla $=71,60+2,54 \times$ Long. Tibia $\pm 2,11$} \\
\hline \multicolumn{2}{|r|}{ Fémur y Tibia: talla $=64,95+1,25 \times$ (Long. Fémur + Long. Tibia $) \pm 2,35$} \\
\hline \multicolumn{2}{|r|}{ Húmero: talla $=99,74+2,19 \times$ Long. Húmero $\pm 3,39$} \\
\hline \multicolumn{2}{|r|}{ Radio: talla $=103,11+2,61 \times$ Long. Radio $\pm 3,20$} \\
\hline \multicolumn{2}{|r|}{ Húmero y Radio: talla $=90,18+1,38 \times$ (Long. Húmero + Long. Radio) $\pm 2,98$} \\
\hline \multicolumn{2}{|r|}{ Ulna: talla $=105,83+2,31 \times$ Long. Ulna $\pm 3,28$} \\
\hline \multicolumn{2}{|r|}{ Fórmulas De Regresión Para Poblaciones Mesoamericanas (Genovés, 1967) } \\
\hline \multirow[t]{2}{*}{ Hombres: } & Fémur: $\quad$ talla $=2,26 \times$ Long. Fémur $+66,379 \pm 3,417$ \\
\hline & Tibia: $\quad$ talla $=1,96 \times$ Long. Tibia $+93.752 \pm 2,815$ \\
\hline \multirow[t]{2}{*}{ Mujeres: } & Fémur: talla $=2,59 \times$ Long. Fémur $+49,742 \pm 3,816$ \\
\hline & Tibia: $\quad$ talla $=2,72 \times$ Long. Tibia $+63,781 \pm 3,513$ \\
\hline \multicolumn{2}{|r|}{ Fórmulas de Regresión para Poblaciones Blancas (Trotter y Gleser, 1958) } \\
\hline \multirow[t]{6}{*}{ Hombres: } & Fémur y Tibia: talla $=1,30$ (Long. Fémur + Long. Tibia) $+63,29$ \\
\hline & Fémur: $\quad$ talla $=2,38 \times$ Long. Fémur $+61,41$ \\
\hline & talla $=2,52 \times$ Long. Tibia $+78,62$ \\
\hline & talla $=3,08 \times$ Long. Húmero $+70,45$ \\
\hline & talla $=3,78 \times$ Long. Radio $+79,01$ \\
\hline & Ulna: $\quad$ talla $=3,70 \times$ Long. Ulna $+74,05$ \\
\hline \multirow{6}{*}{ Mujeres: } & Fémur y Tibia: talla $=1,39 \times$ (Long. Fémur + Long. Tibia $)+53,20$ \\
\hline & Fémur: $\quad$ talla $=2,47 \times$ Long. Fémur $+54,10$ \\
\hline & talla $=2,90 \times$ Long. Tibia $+61,53$ \\
\hline & talla $=3,36 \times$ Long. Húmero $+57,97$ \\
\hline & talla $=4,74 \times$ Long. Radio $+54,93$ \\
\hline & talla $=4,27 \times$ Long. Ulna $+57,76$ \\
\hline \multicolumn{2}{|r|}{ Fórmulas de Regresión para Poblaciones Negras (Trotter y Gleser, 1958) } \\
\hline \multirow[t]{6}{*}{ Hombres: } & Fémur y Tibia: talla $=1,15$ (Long. Fémur + Long. Tibia) $+71,04$ \\
\hline & talla $=2,11 \times$ Long. Fémur $+70,35$ \\
\hline & talla $=2,19 \times$ Long. Tibia $+86,02$ \\
\hline & talla $=3,26 \times$ Long húmero $+61,10$ \\
\hline & talla $=3,42 \times$ Long. Radio $+81,56$ \\
\hline & talla $=3,26 \times$ Long. Ulna $+79,29$ \\
\hline \multirow[t]{6}{*}{ Mujeres: } & Fémur y Tibia: talla $=1,26 \times$ (Long. Fémur + Long. Tibia) $+59,72$ \\
\hline & Fémur: $\quad$ talla $=2,28 \times$ Long. Fémur $+59,76$ \\
\hline & talla $=2,45 \times$ Long. Tibia $+72,65$ \\
\hline & talla $=3,08 \times$ Long. Húmero $+64,67$ \\
\hline & talla $=3,67 \times$ Long. Radio $+71,79$ \\
\hline & talla $=3,31 \times$ Long. Ulna $+75,38$ \\
\hline \multicolumn{2}{|r|}{ Fórmulas de Regresión para poblaciones mongoloides (Trotter y Gleser, 1958) } \\
\hline \multirow[t]{6}{*}{ Hombres: } & Fémur y Tibia: talla $=1,22$ (Long. Fémur + Long. Tibia) $+70,37$ \\
\hline & Fémur: $\quad$ talla $=2,15 \times$ Long. Fémur $+72,57$ \\
\hline & talla $=2,39 \times$ Long. Tibia $+81,45$ \\
\hline & talla $=2,68 \times$ Long. Húmero $+83,19$ \\
\hline & talla $=3,54 \times$ Long. Radio $+82,00$ \\
\hline & talla $=3,48 \times$ Long. Ulna $+77,45$ \\
\hline
\end{tabular}




\begin{tabular}{|c|c|c|c|c|c|c|c|c|c|c|}
\hline \multicolumn{2}{|l|}{ Fórmulas } & OB2 & LM2 & PP1 & $\begin{array}{l}\text { MFM- } \\
2667\end{array}$ & LA2 & PA1 & $\begin{array}{l}\text { MFM- } \\
2670\end{array}$ & $\begin{array}{c}\text { PROM } \\
\text { Masculino }\end{array}$ & $\begin{array}{l}\text { PROM } \\
\text { Femenino }\end{array}$ \\
\hline \multicolumn{2}{|l|}{ Fully } & 155,1 & 170,4 & 156,1 & 170 & 151,7 & 145,7 & 154,1 & 162,9 & 150,5 \\
\hline \multirow{7}{*}{ Béguelin } & $\mathrm{F}$ & 162,6 & 172,6 & 162,2 & 172,0 & 161,0 & 152,3 & 165,6 & 167,4 & 159,6 \\
\hline & $\mathrm{T}$ & 157,2 & 171,3 & 158,5 & 165,3 & 153,6 & 146,8 & 159,2 & 163,1 & 153,2 \\
\hline & $\mathrm{F}+\mathrm{T}$ & 159,8 & 172,4 & 160,2 & 169,1 & 157,1 & 148,8 & 162,5 & 165,4 & 156,2 \\
\hline & $\mathrm{H}$ & 161,9 & 173,5 & 167,6 & 172,2 & 159,3 & 158,7 & 168,0 & 168,8 & 162,0 \\
\hline & $\mathbf{R}$ & 163,7 & 174,9 & 167,1 & 172,0 & 159,5 & 154,0 & 164,7 & 169,4 & 159,4 \\
\hline & $\mathrm{H}+\mathrm{R}$ & 161,4 & 174,6 & 166,8 & 172,3 & 157,5 & 154,2 & 165,7 & 168,8 & 159,2 \\
\hline & U & 164,3 & 173,7 & 167,5 & 171,9 & 159,7 & 157,1 & 165,2 & 169,4 & 160,7 \\
\hline \multirow{2}{*}{ Genovés } & $F$ & 161,8 & 171,9 & 161,3 & 171,3 & 157,1 & 146,9 & 162,5 & 166,6 & 155,5 \\
\hline & $\mathrm{T}$ & 159,8 & 170,7 & 160,8 & 166,1 & 151,6 & 144,3 & 157,6 & 164,3 & 151,2 \\
\hline \multirow{6}{*}{$\begin{array}{c}\text { Trotter y Gleser } \\
\text { Negros }\end{array}$} & $\mathrm{F}$ & 159,4 & 168,9 & 159,0 & 168,3 & 154,3 & 145,3 & 159,1 & 163,9 & 152,9 \\
\hline & $\mathrm{T}$ & 159,8 & 172,0 & 160,9 & 166,8 & 151,8 & 145,2 & 157,2 & 164,9 & 151,4 \\
\hline & $\mathrm{F}+\mathrm{T}$ & 158,3 & 169,9 & 158,7 & 166,9 & 152,6 & 144,3 & 158,1 & 163,4 & 151,7 \\
\hline & H & 154,7 & 172,0 & 163,2 & 170,0 & 148,4 & 147,5 & 160,6 & 165,0 & 152,2 \\
\hline & $\mathrm{R}$ & 160,9 & 175,6 & 165,4 & 171,8 & 151,1 & 143,4 & 158,4 & 168,4 & 150,9 \\
\hline & U & 161,8 & 175,1 & 166,3 & 172,5 & 152,5 & 148,9 & 160,4 & 168,9 & 153,9 \\
\hline \multirow{6}{*}{$\begin{array}{c}\text { Trotter y Gleser } \\
\text { Blancos }\end{array}$} & $F$ & 161,8 & 172,6 & 161,4 & 171,9 & 156,5 & 146,7 & 161,7 & 166,9 & 155,0 \\
\hline & $\mathrm{T}$ & 163,5 & 177,5 & 164,8 & 171,6 & 155,2 & 147,4 & 161,6 & 169,4 & 154,7 \\
\hline & $\mathrm{T}+\mathrm{F}$ & 162,0 & 175,0 & 162,4 & 171,6 & 155,7 & 146,5 & 161,7 & 167,7 & 154,6 \\
\hline & H & 157,9 & 174,2 & 165,9 & 172,4 & 149,4 & 148,4 & 162,6 & 167,6 & 153,5 \\
\hline & $\mathrm{R}$ & 166,7 & 183,0 & 171,6 & 178,8 & 157,3 & 147,4 & 166,8 & 175,0 & 157,2 \\
\hline & U & 167,7 & 182,8 & 172,8 & 179,9 & 157,3 & 152,6 & 167,5 & 175,8 & 159,1 \\
\hline \multirow{6}{*}{$\begin{array}{c}\text { Trotter y Gleser } \\
\text { Mongoloides }\end{array}$} & $\mathrm{F}$ & 163,3 & 173,0 & 162,9 & 172,4 & NC & NC & NC & 167,9 & NC \\
\hline & $\mathrm{T}$ & 162,0 & 175,3 & 163,2 & 169,6 & NC & NC & NC & 167,5 & NC \\
\hline & $T+F$ & 163,0 & 175,2 & 163,3 & 172,0 & NC & NC & NC & 168,4 & NC \\
\hline & $\mathrm{H}$ & 159,3 & 173,5 & 166,3 & 171,9 & $\mathrm{NC}$ & NC & NC & 167,8 & NC \\
\hline & $R$ & 164,1 & 179,4 & 168,7 & 175,5 & NC & NC & NC & 171,9 & NC \\
\hline & U & 165,5 & 179,8 & 170,4 & 177,0 & NC & NC & NC & 173,2 & NC \\
\hline
\end{tabular}

Tabla 3. Estimaciones de la talla $(e n c m)$ según las fórmulas aplicadas. Referencias: T: tibia; F: fémur; OB2: Orejas de Burro 2; LM2: Las Mandíbulas 2; PP1: Puesto Pescador 1; LA2: La Arcillosa 2; PA1: Paiashauaia 1; PROM: promedio; NC: no corresponde.

Table 3. Stature estimated in $\mathrm{cm}$ according to the formulas used. References: T: Tibia; F: Femur; OB2: Orejas de Burro 2; LM2: Las Mandíbulas 2; PP1: Puesto Pescador 1; LA2: La Arcillosa 2; PA1. Paiashanaia 1; PROM: Average; NC: Not aplicable.

y Béguelin (2011) fueron analizados los coeficientes de determinación $\left(r^{2}\right)$, es decir la diferencia para cada individuo entre las estimaciones de talla con la fórmula de Fully y los predichos por la regresión. Este criterio es óptimo para generar modelos explicativos, ya que determina las rectas de regresión lineales bivariadas que se ajustan mejor a los datos. Además, se tuvo en cuenta las posibles diferencias entre individuos de acuerdo con el sexo. En este trabajo no fue posible evaluar la variabilidad espacial de los resultados, debido al reducido número de la muestra analizada.

\section{Resultados}

En la tabla 3 se presentan las estimaciones de talla para los individuos de Patagonia Austral obtenidas a partir de las fórmulas de regresión (Béguelin, 2011; Genovés,
1967; Trotter y Gleser, 1952) de la fórmula anatómica (Raxter et al., 2006) y los promedios por sexo. Cuando estos resultados son analizados a través de la técnica estadística de regresión lineal por mínimos cuadrados, para los individuos masculinos se observa que en 14 de las 27 (51,9\%) fórmulas propuestas por los autores existe un valor de $\mathrm{r}^{2}$ (coeficiente de determinación) igual o superior a 0,9 (Tabla 4). En orden decreciente, la mayoría de las fórmulas evaluadas que requieren la longitud del fémur resultaron en altos valores del coeficiente de determinación en relación con la estimación anatómica de la talla $\left(r^{2} \approx 0,99\right)$, seguidas por todas aquellas que requieren la longitud del fémur y tibia $\left(r^{2} \approx 0,96\right)$. Todas las fórmulas que requieren la longitud de la ulna y las fórmulas de Trotter y Gleser para blancos y mongoloides que emplean la longitud del radio presentaron también altos valores del coeficiente de determinación en relación 
Tabla 4. Resultados obtenidos con la técnica de mínimos cuadrados para los individuos de sexo masculino. Referencia: * valores de $\mathrm{R}^{2}$ considerados significativos respecto de las estimaciones realizadas mediante el método de Fully.

Table 4. Results obtained with the technique of least squares for male individuals. Reference: ${ }^{*} R^{2}$ values considered significant with respect to estimates made using the Fully method.

\begin{tabular}{|l|c|c|}
\hline \multicolumn{1}{|c|}{ Fórmula } & $\mathbf{R}^{\mathbf{2}}$ & Error estándar \\
\hline Béguelin (Fémur) & $0,994^{*}$ & 0,084 \\
\hline Trotter y Gleser Mongoloides (Fémur) & $0,993^{*}$ & 0,088 \\
\hline Trotter y Gleser Negros (Fémur) & $0,993^{*}$ & 0,091 \\
\hline Genovés (Fémur) & $0,993^{*}$ & 0,089 \\
\hline Trotter y Gleser Negros (Fémur + Tibia) & $0,963^{*}$ & 0,195 \\
\hline Trotter y Gleser Blancos (Fémur + Tibia) & $0,962^{*}$ & 0,177 \\
\hline Béguelin (Fémur + Tibia) & $0,962^{*}$ & 0,183 \\
\hline Trotter y Gleser Mongoloides (Fémur + Tibia) & $0,962^{*}$ & 0,188 \\
\hline Trotter y Gleser Blancos (Ulna) & $0,909^{*}$ & 0,263 \\
\hline Béguelin (Ulna) & $0,908^{*}$ & 0,425 \\
\hline Trotter y Gleser Negros (Ulna) & $0,908^{*}$ & 0,302 \\
\hline Trotter y Gleser Mongoloides (Ulna) & $0,905^{*}$ & 0,285 \\
\hline Trotter y Gleser Mongoloides (Radio) & $0,901^{*}$ & 0,274 \\
\hline Trotter y Gleser Blancos (Radio) & $0,900^{*}$ & 0,259 \\
\hline Béguelin (Radio) & 0,898 & 0,382 \\
\hline Trotter y Gleser Negros (Radio) & 0,897 & 0,293 \\
\hline Trotter y Gleser Blancos (Tibia) & 0,874 & 0,077 \\
\hline Trotter y Gleser Blancos (Fémur) & 0,874 & 0,326 \\
\hline Genovés (Tibia) & 0,874 & 0,419 \\
\hline Béguelin (Humero + Radio) & 0,872 & 0,361 \\
\hline Béguelin (Tibia) & 0,871 & 0,328 \\
\hline Trotter y Gleser Negros (Tibia) & 0,871 & 0,379 \\
\hline Trotter y Gleser Mongoloides (Tibia) & 0,870 & 0,350 \\
\hline Trotter y Gleser Blancos (Húmero) & 0,834 & 0,329 \\
\hline Béguelin (Húmero) & 0,833 & 0,464 \\
\hline Trotter y Gleser Negros (Húmero) & 0,832 & 0,313 \\
\hline Trotter y Gleser Mongoloides (Húmero) & 0,832 & 0,381 \\
\hline & & \\
\hline
\end{tabular}

con los resultados obtenidos mediante el método de Fully ( $r^{2} \approx 0,91$ y $r^{2} \approx 0,90$, respectivamente). Por otro lado, las fórmulas que tienen en cuenta la longitud de la tibia resultaron en menores valores del coeficiente de determinación $\left(r^{2} \approx 0,87\right)$, al igual que todas las que requieren la longitud del húmero $\left(r^{2} \approx 0,83\right)$, las fórmulas de Béguelin $\left(r^{2}=0,898\right)$ y de Trotter y Gleser para negros $\left(r^{2}=0,897\right)$ que emplean la longitud del radio, la fórmula de Trotter y Gleser para blancos que requieren la longitud del fémur $\left(r^{2}=0,874\right)$ y la fórmula de Béguelin a partir de la longitud del húmero y radio $\left(r^{2}=0,872\right)$.

En relación con los resultados obtenidos para los individuos femeninos (Tabla 5) se observa que 14 de las $21(66,7 \%)$ fórmulas propuestas resultaron en un valor de $r^{2}$ (nivel de explicación) igual o superior a 0,9. En orden decreciente, también en este caso las fórmulas que requieren la longitud del fémur presentaron las mayores correlaciones respecto del método de Fully $\left(r^{2} \approx 0,99\right)$, seguidas por todas aquellas que emplean la longitud del fémur y tibia $\left(r^{2} \approx 0,98\right)$. A diferencia de lo que ocurre en los individuos masculinos, en los femeninos los resultados muestran altos valores del coeficiente de determinación en las estimaciones realizadas con todas las fórmulas que emplean la longitud de la tibia $\left(r^{2} \approx 0,96\right)$, y la longitud del radio $\left(r^{2} \approx 0,95\right)$. Por debajo del valor de $r^{2}$ menor a 0,9 se encuentran todas las fórmulas que requieren la longitud de la ulna $\left(r^{2} \approx 0,80\right)$, del húmero $\left(r^{2} \approx 0,58\right)$ y la fórmula de Béguelin que tiene en cuenta la longitud del húmero y radio $\left(r^{2}=784\right)$.

\section{Discusión y Conclusiones}

Los estudios de talla en poblaciones antiguas de Patagonia Austral han sido abordados en general en escasas oportunidades, y en todas ellas han empleado el enfoque matemático con fórmulas diferentes construidas a partir de poblaciones que no pertenecen a la misma área (Auerbach y Ruff, 2010; Béguelin, 2011; Feldesman y Fountain, 1996; Trotter y Gleser, 1952). Esto lleva dos problemas concretos: 1) Al no contar con fórmulas de regresión específicamente creadas a partir de individuos de esta región, no se cuenta con información acerca de cuál o cuáles son las más exactas en relación con la talla real. Por lo tanto, la elección de una fórmula de estimación no se basa en información previa, sino más bien en criterios establecidos por cada investigador. 2) Dado que cada estudio empleó formulas diferentes, se desconoce si los resultados obtenidos son comparables entre sí, imposibilitando llegar a conclusiones más extensas.

Los análisis realizados en este trabajo representan la primera tentativa para resolver estas incógnitas. Se enfrenta con la limitación del reducido tamaño muestral, dado que no son frecuentes los esqueletos que cuentan con todos los elementos óseos requeridos para emplear la fórmula anatómica de Fully. Sin embargo, cuando las fórmulas con enfoque matemático son ordenadas de mayor a menor por su valor de $r^{2}$, se agrupan casi perfectamente según el - o los - elementos empleados. De igual modo, la mitad de las fórmulas de estimaciones mostraron altos valores de coeficientes de determinación, en especial en los individuos masculinos, donde las fórmulas indican valores superiores a 0,8 en relación con el método anatómico. Ambos resultados permiten suponer que el tamaño muestral no invalida las conclusiones que puedan obtenerse aquí.

Tanto en esqueletos femeninos como en masculinos, las fórmulas con mayores valores del coeficiente de determinación tienden a agruparse de acuerdo con el o los tipos de huesos requeridos, en general sin distinción de acuerdo con las muestras con las cuales fueron construidas. Es decir, los resultados tendieron a agruparse sin importar la muestra a partir de la cual las fórmulas de regresión fueron construidas (i.e. mongoloides, negros, blancos, mesoamericanos, norte de Patagonia). Las estimaciones de talla más exactas fueron aquellas que 
Tabla 5. Resultados obtenidos con la técnica de mínimos cuadrados para los individuos de sexo femenino. Referencia: * valores de $\mathrm{R}^{2}$ considerados significativos respecto de las estimaciones realizadas mediante el método de Fully.

Table 5. Results obtained with the least squares technique for female individuals. Reference: * $R^{2}$ values considered significant with respect to estimates made using the fully method.

\begin{tabular}{|l|c|c|}
\hline \multicolumn{1}{|c|}{ Formula } & $\mathbf{R}^{\mathbf{2}}$ & Error estándar \\
\hline Béguelin (Fémur) & $0,996^{*}$ & 0,043 \\
\hline Genovés (Fémur) & $0,996^{*}$ & 0,036 \\
\hline Trotter y Gleser Blancos (Fémur) & $0,995^{*}$ & 0,038 \\
\hline Trotter y Gleser Negros (Fémur) & $0,995^{*}$ & 0,042 \\
\hline Béguelin (Fémur + Tibia) & $0,985^{*}$ & 0,076 \\
\hline Trotter y Gleser Blancos (Fémur + Tibia) & $0,985^{*}$ & 0,069 \\
\hline Trotter y Gleser Negros (Fémur + Tibia) & $0,984^{*}$ & 0,078 \\
\hline Trotter y Gleser Negros (Tibia) & $0,966^{*}$ & 0,132 \\
\hline Trotter y Gleser Blancos (Tibia) & $0,966^{*}$ & 0,112 \\
\hline Genovés (Tibia) & $0,966^{*}$ & 0,120 \\
\hline Béguelin (Tibia) & $0,966^{*}$ & 0,129 \\
\hline Béguelin (Radio) & $0,950^{*}$ & 0,181 \\
\hline Trotter y Gleser Negros (Radio) & $0,949^{*}$ & 0,130 \\
\hline Trotter y Gleser Blancos (Radio) & $0,948^{*}$ & 0,102 \\
\hline Béguelin (Ulna) & 0,814 & 0,452 \\
\hline Trotter y Gleser Blancos (Ulna) & 0,809 & 0,248 \\
\hline Trotter y Gleser Negros (Ulna) & 0,807 & 0,323 \\
\hline Béguelin (Humero + Radio) & 0,784 & 0,340 \\
\hline Trotter y Gleser Blancos (Húmero) & 0,582 & 0,353 \\
\hline Trotter y Gleser Negros (Húmero) & 0,580 & 0,383 \\
\hline Béguelin (Húmero) & 0,577 & 0,541 \\
\hline
\end{tabular}

emplean el fémur, tanto en individuos masculinos como en femeninos, con la excepción de la fórmula para el fémur en poblaciones blancas propuesta por Troter y Glesser (1951), la que mostró una valor del coeficiente de determinación inferior a 0,9 respecto de la fórmula de Fully en esqueletos masculinos. Asimismo, resultaron exactas las fórmulas que combinan las longitudes de fémur y la tibia. Las fórmulas desarrolladas por Béguelin (2011) muestran altos valores de coeficientes de determinación en este trabajo, tanto en individuos masculinos como en femeninos. Sin embargo, estas fórmulas están desarrolladas a partir de una muestra de individuos masculinos, por lo que sus resultados en esqueletos femeninos deben ser interpretados con cautela.

Por el contrario, la mayor parte de las estimaciones con las fórmulas que requieren la longitud del humero sobreestimaron la talla de los individuos, lo que se expresa en una baja exactitud en relación con el método anatómico de Fully. Por consiguiente, en esqueletos cuyos miembros inferiores estén ausentes o incompletos, la aplicación de las fórmulas que requieren la longitud de ulna o de radio elaboradas por Trotter y Glesser para mongoloides y blancos podrían representar alternativas exactas. Asimismo, para individuos femeninos podrían ser alternativas adecuadas la aplicación de las fórmulas que requieren la longitud del radio. Sin embargo, los elementos de los miembros superiores pueden estar más sujetos a variaciones de lateralidad asociados a las actividades físicas, lo que podría influir en los resultados.

La dispersión cronológica de la muestra analizada en este trabajo es alta, por lo que no es posible establecer conclusiones acerca de los posibles efectos de los cambios temporales en las estimaciones de la talla de los individuos estudiados aquí. Sin embargo, como se mencionó más arriba, la coherencia observada en los resultados en cuanto a los valores de coeficiente de determinación y la agrupación de fórmulas de acuerdo al elemento empleado hacen suponer que la variación temporal no implica sesgos perceptibles al momento de la elección de una fórmula de regresión para la estimación de la talla.

Como fue destacado, los autores que estimaron la talla en restos humanos de Patagonia Austral en trabajos previos (Hernández et al 1998; Alfonso-Durruty 2017) emplearon diferentes métodos matemáticos de estimación de la talla. Sin embargo, todos ellos incluyeron fórmulas que tienen en cuenta la longitud del fémur. Por lo tanto, según los resultados del presente estudio, podría considerarse que estas estimaciones previas resultan comparables entre sí, aun sin haber arribado a consensos previos.

Las fórmulas identificadas como las más exactas para estimar la talla en esta muestra podrían ser utilizadas en futuros estudios que incluyan conjuntos de restos humanos de Patagonia Austral más numerosos. Esto permitiría evaluar de manera más precisa la variabilidad espacial que ha sido propuesta por varios autores (e.g. Gusinde, 1989; Alfonso Durruty et al., 2017; Cocilovo et al., 2019), la cual es asociada principalmente con su dieta y relaciones parentales. También sería posible evaluar la eventual variabilidad de talla desde el punto de vista temporal, considerando los cambios paleoambientales producidos en la región (Borromei et al., 2007; Haberzettl et al., 2005; Stine, 2000).

Los análisis de la talla a partir de restos humanos son aún escasos en Patagonia Austral en particular, y en Argentina en general. Este hecho contrasta fuertemente con el amplio desarrollo que ha tenido la Bioarqueología y la Paleopatología durante las últimas décadas. Las estimaciones de la talla ofrecen una línea de información potencial acerca del estado de salud de las poblaciones, en especial cuando son analizadas en relación con otras evidencias como la masa corporal o evidencias de estrés sistémico. Este tipo de datos ha sido abundantemente empleado en poblaciones actuales (e.g. Tanner, 1987; Komlos et al., 2007) y en otras poblaciones cazadorasrecolectoras del pasado (e.g. Ousley 1995; Holliday, 1997; Auerbach y Ruff, 2010) alrededor del mundo, por lo que es esperable entonces que los estudios de talla 
sean incorporados en el futuro a las discusiones acerca de la salud de las poblaciones cazadoras-recolectoras que habitaron el sur de Sudamérica.

Ciudad de Buenos Aires, 2 de febrero de 2020

\section{Agradecimientos}

Agradecemos a los Dres. Luis Borrero, Mónica Salemme, Fernando Santiago, Ernesto Piana, Martin Vázquez y Francisco Zangrando, por el acceso a los restos recuperados en el marco de sus respectivos proyectos de investigación, así como a las autoridades del Museo del Fin del Mundo, Lic. Silvia Tale, y al Sr. Lucas Sosa, por su valiosa colaboración. También agradecemos a dos revisores anónimos, cuyos comentarios ayudaron a mejorar considerablemente nuestra investigación. Este trabajo fue financiado por el Proyecto PICT 0191-2016.

\section{Bibliografía}

Alfonso-Durruty, M., Giles, B. T., San Román, M., y Morello, F. (2017). Evaluación de la variación fenotípica del peso, la forma y la masa corporal en grupos cazadores-recolectores prehistóricos de Patagonia y Tierra del Fuego, chile. Chungará (Arica), 49(4), 623634.

Auerbach, B. M. (2011). Methods for estimating missing human skeletal element osteometric dimensions employed in the revised fully technique for estimating stature. Am J Phys Anthropol, 145(1), 67-80. https://doi. org/10.1002/ajpa.21469

Auerbach, B. M., y Ruff, C. B. (2010). Stature estimation formulae for indigenous North American populations. Am J Phys Anthropol, 141(2), 190-207. https://doi. org/10.1002/ajpa.21131

Béguelin, M. (2011). Stature estimation in a central Patagonian prehispanic population: development of new models considering specific body proportions. International Journal of Osteoarchaeology, 21(2), 150-158. https://doi. org/10.1002/oa.1117

Bogin, B. (1999). Patterns of human growth (Vol. 23). Cambridge University Press.

Bogin, B., y Keep, R. (1999). Eight thousand years of economic and political history in Latin America revealed by anthropometry. Ann Hum Biol, 26(4), 333-351. https:// doi.org/10.1080/030144699282651

Borromei, A. M., Coronato, A., Quattrocchio, M., Rabassa, J., Grill, S., y Roig, C. (2007). Late PleistoceneHolocene environments in Valle Carbajal, Tierra del Fuego,
Argentina. Journal of South American Earth Sciences, 23(4), 321-335.

Brooks, S., y Suchey, J. M. (1990). Skeletal age determination based on the os pubis: a comparison of the Acsádi-Nemeskéri and Suchey-Brooks methods. Human evolution, 5(3), 227-238.

Buikstra, J. E., y Ubelaker, D. H. (1994). Standards for data collection from human skeletal remains. Arkansas Archeological Survey Research Series. Fayetteville, AR: Arkansas Archeological Survey.

Cocilovo, J. A., Varela, H. H., y Valdano, S. G. (2019). Hurgando en el arcón de los tiempos perdidos en los confines de la ecúmene. Las etnias selk'nam, yámana y halakwulup según el registro somatométrico de Martín Gusinde (1989 [1937]). InterSecciones en Antropología, 20(1).

Dwight, T. (1894). The Range and Significance of Variation in the Human Skeleton: The Shattuck Lecture for 1894. The Boston Medical and Surgical Journal, 131(4), 73-76.

Feldesman, M. R., y Fountain, R. L. (1996). "Race" specificity and the femur/stature ratio. Am J Phys Anthropol, 100(2), 207-224.

Fully, G. (1956). Une nouvelle méthode de détermination de la taille. Annales de Médicine Légale et de Criminologie, 36, 266-273.

Genovés, S. (1967). Proportionality of the long bones and their relation to stature among Mesoamericans. Am J Phys Anthropol, 26(1), 67-77.

Guichón, R. A., Muñoz, A. S., y Borrero, L. A. (2000). Datos para una tafonomía de restos óseos humanos en Bahía San Sebastián, Tierra del Fuego. Relaciones de la Sociedad Argentina de Antropología, 25.

Gusinde, M. (1989). Tomo 4: Antropología Física (H. H. R. Wolfang J., Carlota, Trans.). In Los indios de Tierra del Fuego. CAEA.

Haberzettl, T., Fey, M., Lücke, A., Maidana, N., Mayr, C., Ohlendorf, C., Schäbitz, F., Schleser, G. H., Wille, M., y Zolitschka, B. (2005). Climatically induced lake level changes during the last two millennia as reflected in sediments of Laguna Potrok Aike, southern Patagonia (Santa Cruz, Argentina). Journal of Paleolimnology, 33(3), 283-302.

Hernández, M., García-Moro, C., y Lalueza-Fox, C. (1998). Stature estimation in extinct Aónikenk and the myth of Patagonian gigantism. Am J Phys Anthropol, 105(4), 545-551. 
Holliday, T. W. (1997). Body proportions in Late Pleistocene Europe and modern human origins. Journal of Human Evolution, 32(5), 423-448. https://doi.org/10.1006/ jhev.1996.0111

Holliday, T. W., y Ruff, C. B. (1997). Ecogeographical patterning and stature prediction in fossil hominids: Comment on MR Feldesman and RL Fountain, American Journal of Physical Anthropology (1996) 100: 207-224. Am J Phys Anthropol, 103(1), 137140.

Hoppa, R. D., y Fitzgerald, C. M. (1999). Human growth in the past: studies from bones and teeth (Vol. 25). Cambridge University Press.

King, S. E., y Ulijaszek, S. J. (1999). Invisible insults during growth and development: contemporary theories and past populations. In R. D. Hoppa y C. M. FitzGerald (Eds.), Human growth in the past: studies from bones and teeth (pp. 161-182). Cambridge University Press.

Komlos, J. (1998). Shrinking in a growing economy? The mystery of physical stature during the industrial revolution. The Journal of Economic History, 58(3), 779802.

Komlos, J., y Lauderdale, B. E. (2007). The mysterious trend in American heights in the 20th century. Annals of human biology, 34(2), 206-215.

Larsen, C. S. (1997). Bioarchaeology: interpreting behavior from the human skeleton. Cambridge University Press.

Lovejoy, C. O., Meindl, R. S., Pryzbeck, T. R., y Mensforth, R. P. (1985). Chronological metamorphosis of the auricular surface of the ilium: a new method for the determination of adult skeletal age at death. Am J Phys Anthropol, 68(1), 15-28.

Lundy, J. K. (1985). The mathematical versus anatomical methods of stature estimate from long bones. Am J Forensic Med Pathol, 6(1), 73-76. https://doi. org/10.1097/00000433-198503000-00013

Neves, W. A., y Costa, M. A. (1998). Adult stature and standard of living in the prehistoric Atacama Desert. Current Anthropology, 39(2), 278-281.

Olivier, G. (1969). Practical anthropology. Charles C Thomas.

Ousley, S. D. (1995). Relationships between Eskimos, Amerindians, and Aleuts: Old data, new perspectives. Human biology, 67(3), 427-458.

Pearson, K. (1899). IV. Mathematical contributions to the theory of evolution. $-\mathrm{V}$. On the reconstruction of the stature of prehistoric races. Philosophical Transactions of the Royal Society of London. Series A, Containing Papers of a Mathematical or Physical Character(192), 169-244.

Pomeroy, E., y Stock, J. T. (2012). Estimation of stature and body mass from the skeleton among coastal and mid-altitude Andean populations. Am J Phys Anthropol, 147(2), 264-279. https://doi.org/10.1002/ ajpa. 21644

Raxter, M. H., Auerbach, B. M., y Ruff, C. B. (2006). Revision of the Fully technique for estimating statures. Am J Phys Anthropol, 130(3), 374-384. https://doi. org/10.1002/ajpa.20361

Rollet, É. (1888). De la mensuration des os longs des membres dans ses rapports avec I'anthropologie, la clinique et la médecine judiciaire. G. Steinheil.

Santiago, F., Salemme, M., Suby, J., Guichón, R. (2011). Restos humanos en el Norte de Tierra del Fuego: aspectos contextuales, dietarios y paleopatológicos. InterSecciones en Antropología, 12(1), 147-162.

Saunders, S. R., y Hoppa, R. D. (1993). Growth deficit in survivors and non-survivors: biological mortality bias in subadult skeletal samples. Am J Phys Anthropol, 36(S17), 127-151.

Soto-Heim, P. (2001). Considérations sur la stature des populations sub-actuelles de Patagonie et Extrême Sud d'Amerique. Biométrie humaine et anthropologie, 19(34), 257-264.

Stine, S. (2000). On the medieval climatic anomaly. Current Anthropology, 41(4), 627-628.

Stinson, S. (1990). Variation in body size and shape among South American Indians. American Journal of Human Biology, 2(1), 37-51.

Suby, J. A., Santiago, F., y Salemme, M. (2008). Análisis paleopatológico de los restos humanos del sitio Puesto Pescador 1 (Tierra del Fuego). Magallania (Punta Arenas), 36(1), 53-64.

Tanner, J. M. (1987). Issues and advances in adolescent growth and development. Journal of Adolescent Health Care, 8(6), 470-478. https://www.ncbi.nlm.nih.gov/ pubmed/3121548

Todd, T. (1921a). Age changes in the pubic bone. I: the male white pubis. Am J Phys Anthropol, 3, 285-334.

Todd, T. (1921b). Age changes in the pubic bone. III: 
the pubis of the white female. IV: the pubis of the female white-negro hybrid. Am J Phys Anthropol, 4, $1-70$.

Trotter, M., y Gleser, G. C. (1952). Estimation of stature from long bones of American Whites and Negroes. Am J Phys Anthropol, 10(4), 463-514. http://www.ncbi.nlm. nih.gov/pubmed/13007782

Varela, H. H., Cocilovo, J. A., y Guichón, R. (1993-94).
Evaluación de la información somatométrica por Gusinde sobre los aborígenes de Tierra del Fuego. Ans. Inst. Pat. Ser. Cs. Hs, 22, 193-205.

Vercellotti, G., Piperata, B. A., Agnew, A. M., Wilson, W. M., Dufour, D. L., Reina, J. C., Boano, R., Justus, H. M., Larsen, C. S., y Stout, S. D. (2014). Exploring the multidimensionality of stature variation in the past through comparisons of archaeological and living populations. Am J Phys Anthropol, 155(2), 229-242. 